\title{
Surface erosion and modification by highly charged ions
}

\author{
Z. Insepov, ${ }^{1,2, *}$ M. Terasawa, ${ }^{2,3}$ and K. Takayama ${ }^{2}$ \\ ${ }^{1}$ Argonne National Laboratory, 9700 South Cass Avenue, Argonne, Illinois 60439, USA \\ ${ }^{2}$ High Energy Accelerator Research Organization (KEK), 1-1 Oho, Tsukuba 305-0801, Japan \\ ${ }^{3}$ LASTI, University of Hyogo, 3-1-2 Kamigori-cho, Hyogo 678-1205, Japan
}

(Received 11 October 2007; revised manuscript received 15 February 2008; published 9 June 2008)

\begin{abstract}
Analyses were conducted of various models and mechanisms of highly charged ion (HCI) and swift-heavy ion energy transfer into a solid target, such as hollow atom formation, charge screening, neutralization, shock wave generation, crater formation, and sputtering. A plasma model of space charge neutralization based on impact ionization of semiconductors at high electric fields was developed and applied to analyze HCI impacts on Si and W. Surface erosions of semiconductor and metal surfaces caused by HCI bombardments were studied by using a molecular dynamics simulation method, and the results were compared with experimental sputtering data.

DOI: 10.1103/PhysRevA.77.062901

PACS number(s): 79.20.Rf, 71.15.Pd, 34.50.Fa, 61.80.Az
\end{abstract}

\section{INTRODUCTION}

Interactions of energetic ions, such as highly charged ions (HCIs), swift heavy ions (SHIs), and fission debris with solid surfaces and bulk materials have fundamental and practical interest in such areas as magnetic storage [1], latent track formation [2], multiple ionization in solid targets [3-7], spallation neutron sources [8], extreme ultraviolet lithography source development [9], HCI-driven secondary ion mass spectrometry for surface analysis [10], and protein desorption by HCI impacts [11].

$\mathrm{HCI}$ impacts on semiconductor and insulator targets have many similarities to the impacts of SHIs with solid targets. As occurs at an impact of a swift ion with an insulating surface, the sputtering yield of $\mathrm{HCI}$ is significantly higher than that predicted by the linear sputtering theory [12] and cannot be understood with any of the existing theoretical models [13]. The potential energy of the colliding HCI is transferred into the electronic degrees of freedom of the target.

A plasma model of the swift ion interaction with surfaces developed in [6] predicts that a track core is formed in $\tau_{0}$ $\sim 10^{-17} \mathrm{~s}$. The track core is formed by the heavy ion collisions with the target's atoms at a small impact parameter. The average diameter of the core is about $1 \AA$. During this process, $\delta$ electrons are ejected, and excited states are created. A strong electric field attracts the emitted $\delta$ electrons and returns them to the core region within $\tau_{1} \sim 10^{-15}$ $-10^{-14} \mathrm{~s}$. The core region ions start expanding as a result of Coulomb interaction during this stage and obtain kinetic energy in $\tau_{2} \leq 10^{-13} \mathrm{~s}$. The core atoms are excited and are expected to have long lifetimes, $\tau_{3} \sim 10^{-9} \mathrm{~s}$. The hot electronic system eventually transfers the energy into the cold ionic system. The plasma model [6] also predicts that a large electric field $\sim 10^{6} \mathrm{~V} / \mathrm{cm}$ may develop at a distance of $5 \AA$ from the track of a $1 \mathrm{MeV} \mathrm{He}^{+}$ion in frozen argon.

Two other models, a simple Maxwell charge neutralization model and a hollow atom (HA) formation model, also

\footnotetext{
*insepov@anl.gov, FAX: 1-630-252-5986.
}

have been widely used to characterize materials properties in response to highly charged ions. The Maxwell charge neutralization model defines the space charge relaxation based on the local conductivity. The HA model is used to study the relaxation of HCI approaching a metal or semiconductor surface.

In this paper we describe the adaptations made to all three models in order to study erosion of semiconductor and metal surfaces irradiated with highly charged ions. These models were then used to provide input parameters for our simulations of surface erosion and modification. At the heart of our simulations is molecular dynamics (MD). This powerful theoretical method has been used by researchers to calculate important energetic ion-surface collision characteristics, such as shock wave generation, crater formation, sputtering and reflection yields, sticking probabilities of ions on various surfaces, and substrate temperature effects [13]. MD has also been used to investigate thermodynamics and kinetics of solid-state plasma generated by powerful laser or energetic heavy-ion interaction with solid targets [7]. Here we use MD techniques, in combination with the three cited models, to simulate Coulomb explosion on semiconductor surfaces. Silicon and tungsten substrates were chosen as important high- $Z$ materials for future fusion, extreme ultraviolet (EUV) lithography, and heavy-ion fusion device developments.

Simulation results for silicon and tungsten sputtering show good agreement with experiment. Moreover, the simulated crater formation with HCI ion bombardment shows a smaller diameter than the experimental value, with a clean crater and smaller rim. These results are promising for future applications such as wafer etching in the semiconductor and magnetic storage industries.

\section{SIMULATION MODELS}

This section details the three models that were used as input for the molecular dynamics simulations. Described first is a hollow atom (HA) formation model, which was used to study the dynamics of HA formation. This description is followed by descriptions of two models used to obtain characteristic neutralization times: (1) a simple Maxwell charge 


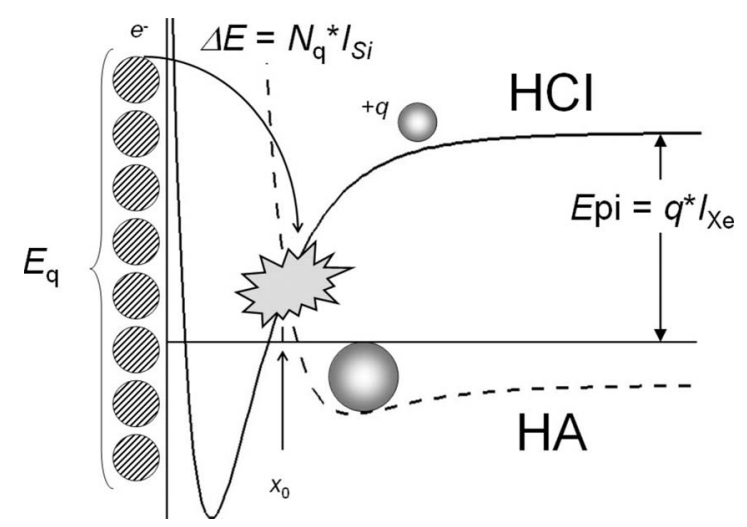

FIG. 1. Physical model of hollow atom formation based on the classical over-the-barrier model [14].

neutralization model which defines the space charge relaxation based on the local conductivity; and (2) a plasma model, which evaluates space charge neutralization by using a set of equations for carrier mobility and annihilation, ambipolar diffusion, and impact ionization of the target. The neutralization times were then incorporated into a molecular dynamics simulation code in such a way that the total number of charges was adjusted to a radioactive decay law, with the times obtained in the neutralization models.

\section{A. Electronic model of hollow atom formation}

A widely used model for studying the relaxation (neutralization) of HCI approaching a metal or semiconductor surface gives the following scenario [14]. The strong Coulomb field of HCI can pull the electrons from the solid surface. The electrons are then captured into Rydberg states of the ion by the mechanism of resonant capture. Thus a superexcited state, the so-called hollow atom, is formed, which evolves further by emitting electrons and/or photons via the Auger processes. ${ }^{1}$

The potential energy of $\mathrm{Xe}^{q+}(q \leq 54)$ is calculated by a multiconfiguration Dirac-Fock method [15]. Formation of the HA was modeled by switching the interaction potential of the Xe ion with the surface atoms. The dynamics of HA formation was studied via visualization of the events by recording movies at various energies and charge states $q$.

Figure 1 shows a physical model of HA formation. The potential function of a highly charged ion is shown as a solid curve HCI. The total energy of HCI is roughly equal to the total ionization energy: $E_{\mathrm{pi}}=q I_{\mathrm{Xe}}$, where $q$ is the charge state. HA depicts the potential function of a hollow atom. The classical over-the-barrier (COB) model $[14,16]$ is widely used to estimate the distance where the first resonant charge transfer can take place. Resonant neutralization of HCI occurs at a distance $x_{0}$ where two potential curves cross each other.

\footnotetext{
${ }^{1}$ We consider HA of the "first generation," which exist only above the surface (see, e.g., Ref. [13]).
}

$$
x_{0}=\sqrt{\frac{2 q}{W}},
$$

where $W$ is the work function of the surface and $q$ is the charge state of $\mathrm{HCI}^{2}{ }^{2}$ This formula gives the following estimate for the distance above a flat metal surface where $\mathrm{HCI}$ is neutralized: $x_{0} \sim 20 \AA$.

The HA lifetime is much greater than the interaction time of HA with the surface, which is on the order of $\tau_{I}$ $\sim 10^{-13} \mathrm{~s}$ [17]. The HA formation is based on the COB model $[14,16]$. We assume that the processes of charge capture from the surface and electric field screening inside the target are much faster than those that lead to surface sputtering and crater formation. A similar approach is used in Ref. [18].

\section{B. Simple model of space charge neutralization}

In this section, a simple charge neutralization model is developed that allows one to calculate a characteristic time for charge neutralization analytically, by using the material properties. The total number of electrons pulled out of the solid surface can be greater than the initial charge of the ion and is controlled by the total energy conservation law. If the process of HCI relaxation is fast enough, a highly charged zone is formed in close proximity to or "below" the falling ion. Strong repulsive interaction between the newly formed ions belonging to the target produces the so-called Coulomb explosion effect, which in turn leads to formation of a nanocrater on the surface and an enhancement in sputtering.

According to the thermodynamics of ion-electron plasma, an excessive charge with the density $\rho$ inserted into a plasma with the volume $V$ will be neutralized within a characteristic time called the Maxwell relaxation time. This time can be obtained by solving the static Maxwell equations for the electric field $\boldsymbol{E}$, current density $\boldsymbol{J}$, and conductivity $\sigma$ as follows:

$$
\begin{gathered}
\operatorname{div} \boldsymbol{E}=\rho / \varepsilon \varepsilon_{0}=e \Delta n / \varepsilon \varepsilon_{0}, \\
\operatorname{div} \boldsymbol{J}=-\partial \rho / \partial t, \\
\boldsymbol{J}=\boldsymbol{\sigma} \boldsymbol{E}
\end{gathered}
$$

In a one-dimensional (1D) case, the solution of Eq. (2) can be found analytically:

$$
\begin{gathered}
N_{q}(t)=N(0) \exp (-t / \tau), \\
\tau=\varepsilon \varepsilon_{0} / \sigma .
\end{gathered}
$$

Here, $N_{q}(t)=\rho V$, the total number of charges at a time $t$, and $\varepsilon$ and $\varepsilon_{0}$ are the electrical permittivities of a material and vacuum, respectively. The neutralization time has the following meaning: If this time is passed after the charge was introduced into a plasma, the electrical potential of the charge is screened by a Debye-Hückel field.

\footnotetext{
${ }^{2}$ All variables here and further are given in atomic units, unless stated otherwise.
} 
TABLE I. Neutralization times of various materials obtained from the Maxwell relaxation formula given in the text. Conductivity of $\mathrm{LiF}$ was not used in this paper and is given here for comparison only.

\begin{tabular}{ccc}
\hline \hline Materials & $\begin{array}{c}\text { Conductivity, } \\
\left(\Omega^{*} \mathrm{~m}\right)^{-1}\end{array}$ & $\begin{array}{c}\text { Neutralization } \\
\text { time, } \tau \\
(\mathrm{fs})\end{array}$ \\
\hline $\mathrm{Cu}$ & $5.88 \times 10^{7}$ & 0.01 \\
$\mathrm{Al}$ & $3.77 \times 10^{7}$ & 0.024 \\
$\mathrm{~W}$ & $1.89 \times 10^{7}$ & 0.1 \\
$\mathrm{Ni}$ & $1.43 \times 10^{7}$ & 0.13 \\
$\mathrm{Si}$ & 100 & $10^{3}$ \\
$\mathrm{LiF}$ & $10^{-4}$ & $10^{9}$ \\
\hline \hline
\end{tabular}

A formula similar to Eq. (3) was proposed in Ref. [18], where the neutralization times were obtained from experiments, without referring them to the Maxwell relaxation time. A simple plasma relaxation model proposed here by Eq. (4) allows one to find the neutralization times directly from the fundamental properties of materials. Table I shows the times calculated by using formula (4). ${ }^{3}$

It is known that an $\mathrm{HCI}$ is neutralized very rapidly $(<10 \mathrm{fs})$ in solids [15]. Therefore the electronic potential energy of HCI (the energy needed to strip the atomic electrons to the charge state $q+$ of the $\mathrm{HCI}$ ) is released very near the surface. The higher the charge of the ion, the more effect it produces on the surface during bombardment.

Two important physical effects were taken into account: charge neutralization and electric field screening. These two effects are closely related. Specifically, the screened electrical potential is applied to charges after the neutralization time has passed.

The characteristic charge neutralization time was approximated by the Maxwell relaxation time, which gives $\tau_{n}$ $\approx 1 \mathrm{ps}$ for $\mathrm{Si}$. Therefore the charge neutralization effect in $\mathrm{Si}$ is less important because this time is much longer than the interaction and erosion times. However, these times are much shorter for conductive targets. For example, tungsten has a time of $0.1 \mathrm{fs}$, copper and gold $0.02 \mathrm{fs}$. Therefore any atomistic simulation model of HCI interaction with conductive targets should treat the charge neutralization dynamically, for example, by simultaneously solving the Poisson equation for the electrons in the target.

The algorithm of neutralization was used where the ions are sorted according to their $z$-axis positions. For the "neutralization" a Monte Carlo algorithm picked first those ions that were located at the bottom of the hemispherical volume (light circles) as shown in Fig. 2(a). Figure 2(b) shows a typical evolution of the total numbers of charges inside the hot spot volume on the surface of the target for two ion energies and two relaxation times. The initial value of the total number of charges was obtained by using the energy conservation, and then calculated each time step in MD.

The charge "neutralization" procedure was implemented dynamically, that is, during the run of the main MD code.

\footnotetext{
${ }^{3}$ We used this formula for metals with $\varepsilon=\varepsilon_{\infty}$.
}
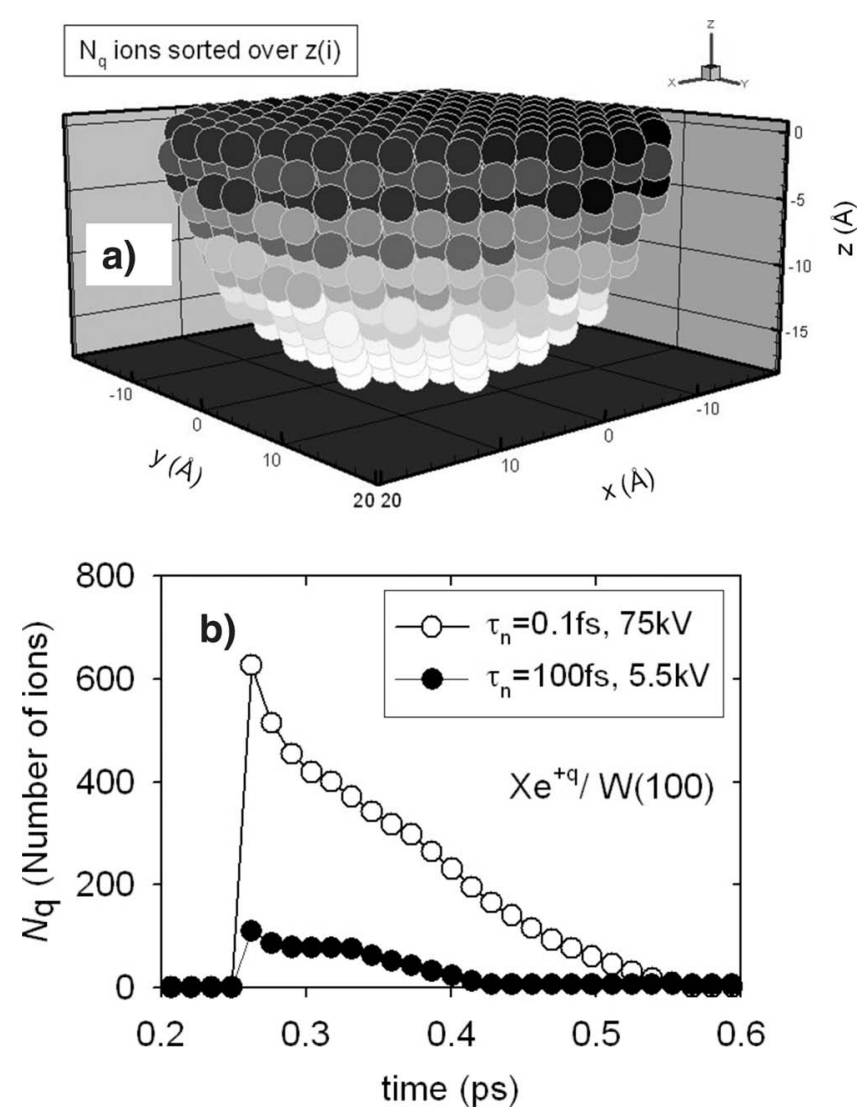

FIG. 2. (a) Simulation model of space charge neutralization: the ions are sorted according to their $z$-axis positions and a Monte Carlo algorithm of the neutralization process was used to pick up for "the neutralization act" the candidate ions that were located near the bottom of the hemispherical volume (light circles were picked up first). (b) The total number of charges calculated by MD simulation. The initial charge was obtained by the energy conservation Eq. (2). The evolution of this variable was modeled by a Monte Carlo module within the MD code.

The ions inside the charged area [Fig. 2(a)] were dynamically sorted according to their depth positions in the target. Each ion belonging to the charged semispherical volume was assigned an individual clock variable. During the run of the MD program, the individual clock variable was compared to the MD time variable at each time step, by using a Monte Carlo (MC) procedure. If the ionic clock variable exceeds the MD time, the MC procedure switches off the ion interaction, switches on the neutral atom interaction, and reassigns the ions in the system by using a probability obtained by the neutralization time and by the location radius of the ion. This procedure reflects the fact that those ions that are located more deeply inside the target should be neutralized faster than those that are located closer to the surface. The total number of space charges (ions) in this approach is a function of time and is shown in Fig. 2(b) for two HCI impacts with different energies, $5.5 \mathrm{keV}$ (solid circles) and $75 \mathrm{keV}$ (open circles).

\section{Plasma model of space charge neutralization}

The plasma model of track formation by a swift heavy ion proposed in Ref. [6] was applied to HCI impacts on semi- 
TABLE II. Electron and hole impact ionization rates, which satisfy the Chynoweth's law [20,21]: $\alpha(E)$ $=\alpha_{\infty} \exp (-b /|E|), \mathrm{cm}^{-1}$.

\begin{tabular}{ccc}
\hline \hline $\begin{array}{c}\text { Electron ionization } \\
\text { rate }\end{array}$ & \multicolumn{2}{c}{ Hole ionization rates } \\
\cline { 2 - 3 } & $1.75 \times 10^{5} \leq E \leq 4 \times 10^{5} \mathrm{~V} / \mathrm{cm}$ & $4 \times 10^{5} \leq E \leq 6 \times 10^{5} \mathrm{~V} / \mathrm{cm}$ \\
\hline$\alpha_{\infty}=7.03 \times 10^{5} \mathrm{~cm}^{-1}$ & $\alpha_{\infty}=1.582 \times 10^{6} \mathrm{~cm}^{-1}$ & $\alpha_{\infty}=6.71 \times 10^{5} \mathrm{~cm}^{-1}$ \\
$b=1.231 \times 10^{6} \mathrm{~V} / \mathrm{cm}$ & $b=2.036 \times 10^{6} \mathrm{~V} / \mathrm{cm}$ & $b=1.693 \times 10^{6} \mathrm{~V} / \mathrm{cm}$ \\
\hline \hline
\end{tabular}

conducting surfaces. This model was further developed by adding the following effects: impact ionization by a high electric field, space charge accumulation, ambipolar diffusion of carriers, conductive drift in the space, and annihilation of electrons and holes. The ionization in high electric fields is a nonlinear effect that cannot be studied by a linear charge neutralization model. The strong electric field generated by HCI impacts on solids is quantitatively evaluated.

Although the model predicts the importance of the high electric fields generated in a $\mathrm{HCI}$ collision and can predict the charge neutralization times, no attempts have been made to directly incorporate it into the MD scheme because there are still many unknown parameters such as the initial core structure and hole parameters.

Space charge neutralization due to $\mathrm{HCI}$ impact on a surface is a complex task involving drift and ambipolar diffusion of two or more types of charge carriers. Therefore the neutralization time of the space charge should be obtained as a solution of the set of equations that contain drift diffusion, Auger transitions, and lattice and impurity relaxation terms; this will be the subject of a separate study. Here we focus on charge neutralization at a $\mathrm{HCI}$ impact based on a simplified model.

The electron, hole, and core hole ${ }^{4}$ fluxes are defined by the diffusion and drift in an electric field $\boldsymbol{E}$ of the space charge [19],

$$
\begin{gathered}
\mathbf{J}_{e}=e D_{e} \boldsymbol{\nabla} N_{e}+e \mu_{e} N_{e} \mathbf{E}, \\
\mathbf{J}_{h}=-e D_{h} \boldsymbol{\nabla} N_{h}+e \mu_{h} N_{h} \mathbf{E}, \\
\mathbf{J}_{\mathrm{ch}}=-e D_{\mathrm{ch}} \boldsymbol{\nabla} N_{\mathrm{ch}}+e \mu_{\mathrm{ch}} N_{\mathrm{ch}} \mathbf{E},
\end{gathered}
$$

where $e$ is the elemental charge amount $(e>0), D_{e, h}$ are the diffusion coefficients, and $\mu_{e, h}$ are the mobilities of the intrinsic electrons and holes at temperature $T$ created by impact ionization in the space charge field. The Einstein relationship between the diffusion coefficients and the mobilities $D_{e, h}$ $=\mu_{e, h} k_{B} T / e$ allows us to use the electric-field-dependent diffusion coefficients; $N_{e, h}$ are the corresponding carrier densities. The third equation for the flux $J_{\text {ch }}$ in the system (5) belongs to core holes, namely, to vacancies in $K$ and $L$ shells of target atoms.

By using Eq. (5), one can write the continuity equations for the electron-hole plasma system in the following form [19]:

\footnotetext{
${ }^{4} K$ - and $L$-shell vacancies.
}

$$
\begin{aligned}
\frac{\partial N_{e}}{\partial t}= & \frac{1}{q} \nabla J_{e}+G_{\mathrm{ii}}=D_{e} \Delta N_{e}+e N_{e} \mu_{e} / \varepsilon \varepsilon_{0}\left(N_{h}+N_{c h}-N_{e}\right) \\
& +G_{\mathrm{ii}} \\
\frac{\partial N_{h}}{\partial t}= & -\frac{1}{q} \nabla J_{h}+G_{\mathrm{ii}}=D_{h} \Delta N_{h}-e N_{h} \mu_{h} / \varepsilon \varepsilon_{0}\left(N_{h}+N_{\mathrm{ch}}-N_{e}\right) \\
& +N_{\mathrm{ch}} / \tau_{\text {Auger }}+G_{\mathrm{ii}}, \\
\frac{\partial N_{\mathrm{ch}}}{\partial t}= & -\frac{1}{q} \nabla J_{\mathrm{ch}}=D_{\mathrm{ch}} \Delta N_{\mathrm{ch}}-e N_{\mathrm{ch}} \mu_{\mathrm{ch}} / \varepsilon \varepsilon_{0}\left(N_{h}+N_{\mathrm{ch}}-N_{e}\right) \\
& -N_{\mathrm{ch}} / \tau_{\text {Auger }} .
\end{aligned}
$$

Here $\tau_{\text {Auger }}$ is the Auger transition time, and $G_{\text {ii }}$ is the rate of impact ionization given by the Chynoweth law [20]

$$
G_{\mathrm{ii}}=\alpha_{n} \frac{J_{e}}{e}+\left(\alpha_{h} \frac{J_{h}}{e}+\alpha_{\mathrm{ch}} \frac{J_{\mathrm{ch}}}{e}\right),
$$

where the coefficients $\alpha_{n}, \alpha_{h}$ for the impact ionization rates are given in Ref. [21] and are shown in Table II.

$$
\begin{gathered}
N_{1}^{2}=N_{c} N_{v} \exp \left(-E_{g} / k_{B} T\right), \\
N_{c} N_{v}=2\left(2 \pi k T / h^{2}\right)^{3 / 2}\left(m_{e} m_{h}\right)^{3 / 4}, \\
G_{\mathrm{ii}}=\alpha_{n} \frac{J_{e}}{e}+\left(\alpha_{h} \frac{J_{h}}{e}+\alpha_{\mathrm{ch}} \frac{J_{\mathrm{ch}}}{e}\right), \\
\Delta^{2} \phi=\frac{e}{\varepsilon \varepsilon_{0}}\left(N_{e}-N_{h}-N_{c h}\right) .
\end{gathered}
$$

$N_{c, v}$ are the effective densities of states in the conductance and valence bands, respectively; $N_{i}$ is the intrinsic carrier density at temperature $T ; k_{B}$ is the Boltzmann constant; $E_{g}$ is the band gap; $m_{e, h}$ are the effective carrier masses; $h$ is the Planck constant; and $\varepsilon_{0}$ and $\varepsilon$ are the permittivities of vacuum and silicon, respectively. The last equation in Eq. (8) is the Poisson equation for the electric field and potential $\phi$ : $\boldsymbol{E}=-\operatorname{grad} \phi$ of the plasma containing the space charge, with the density $N_{\mathrm{ch}}$, and the carrier charges with the densities $N_{e, h}$.

The transitions between the bound and free electron states are well-studied in a conventional plasma $[21,22]$. However, the solid-state plasma generated by a HCI impact in a solid target is much more complex than the gas plasma. The solidstate plasma contains the core hole (ch) states, for example, the $K$ and $L$ shell vacancies created by the process of hol- 
low atom formation; free carriers $(e, h)$ intrinsically existing in a semiconductor as a result of thermal excitation; Auger carriers; and the charges generated by impact ionization in a strong electric field, which are difficult to evaluate. The carrier-phonon and carrier-impurity relaxation terms are usually much slower for indirect semiconductors than the neutralization time and thus can be neglected.

Therefore in our preliminary study we simplify the processes by assuming that there exist electrons and two types of holes: heavy ones that are induced by HCI impact; and light ones that are intrinsic or generated by impact ionization. The transition between the heavy and light holes occurs with the times characteristic for the Auger transition processes.

\section{Molecular dynamics}

Molecular dynamics has been widely used before to calculate temperature, pressure, and energy of planar (1D) steady-state shock waves [23-36] to determine the velocity of a surface shock wave due to ion impact [27], to simulate a shock wave generation within a cluster [28], and to study cluster impacts [29-31].

In the present paper, the dynamics of energetic HCI impacts on $\mathrm{Si}$ and $\mathrm{W}$ (100) surfaces were examined by using a molecular dynamics method that was coupled with a charge neutralization model [18]. The boundary conditions were the same as those used in our previous paper [37], where a multiscale molecular dynamics method was presented. This method combines conventional atomistic MD for the central cluster collisional zone with a continuum mechanics representation for the rest of a system. It significantly reduces the system size and can keep the accuracy of the energy flow through the system boundaries.

The basic MD cell was divided into spherical layers of width $d r$; and the local target variables such as temperature, pressure, energy, and the velocity of moving matter (mass velocity) within a spherical layer were calculated. Local target temperatures were obtained from the equipartition theorem by deducting atomic kinetic energies from the average kinetic energy for the given spherical layer. Local pressures were calculated from a virial formula $[23,24,38]$.

A shock wave front in an ideal nonviscous and a nonthermal conductive gas is a zero-thickness surface that moves with a hypersonic velocity. In a real solid it has a certain thickness defined by a real material viscosity and thermal conductivity [39]. At a shock-front, the local temperature, pressure, and energy acquire an abrupt increase from their equilibrium values before the front, for example, room temperature and zero pressure, to much higher values behind the front. In a classical (macroscopic) shock, the pressure, volume (or density), and temperature in front of and behind the wave are related through a simple formula known as Hugoniot's relation, which represents mass, momentum, and energy conservation laws [39].

The atomic-scale shock wave emerging from the cluster impact has been obtained as a steep increase of radial and transversal kinetic energies of the target atoms according to the technique described above for which a spherical layer thickness $d r=3 \AA$ was used as in [23,24]. The front of this rise has been considered as a shock wave front. This definition of a shock wave front was used in $[25,26]$ for a planar shock.

\section{E. Surface erosion of solid targets}

MD models of surface sputtering were developed for various materials that included $\mathrm{Si}, \mathrm{W}$, and $\mathrm{Nb}$ and for various energy regions. The Stillinger-Weber and Born-Mayer potential functions were used for $\mathrm{Si}[40,41]$ and a Finnis-Sinclair potential for bcc tungsten [42].

The surface slab was bombarded by HCIs $\mathrm{Xe}^{q+}$ where the charge state was varied $q=8-44$. The dynamics of particle ejection (sputtering) from the surface and crater formation on the surface by a HCI impact were simulated. The particles representing the charged zone on the surface were placed inside the hemisphere with its equator lying on the upper plane of the sample. The number of ions $N_{q}$ is computed by the MD method so that the total potential energy of ions $\left(E_{q}\right)$ embedded in a hemispherical region plus the ionization energy of $N_{q}$ ions should be equal to the potential energy of the incident $\mathrm{Xe}^{q+}\left(E_{\mathrm{pi}}\right)$.

The energy balance gives the following relation between the HCI potential energy $E_{\mathrm{pi}}$ and the potential energy of the fully singly ionized volume in the substrate, $E_{q}$ :

$$
E_{\mathrm{pi}}=E_{q}+N_{q} I_{\mathrm{Si}}+E_{\mathrm{se}}+E_{\mathrm{ph}},
$$

where $N_{q}$ and $I_{\mathrm{si}}$ are the number of $\mathrm{Si}^{+}$ions created during the HA formation and the ionization energy of a $\mathrm{Si}$ atom, respectively, and $E_{\mathrm{se}}$ and $E_{\mathrm{ph}}$ are the energies spent for the generation of secondary electrons and photons, respectively.

The sputtering yields as a function of the potential energy of $\mathrm{Xe}^{q+}$ were studied. We obtained this value as a long-time limit of a function $y(t)$, which represents the total number of atoms that crossed a certain control plane at a height $z_{\text {cut }}$ above the surface, with $z_{\text {cut }}$ taken as a parameter. The value of $z_{\text {cut }}=2 R_{\text {cut }}$ was chosen, where $R_{\text {cut }}$ is the cutoff distance for the interaction potential. The atoms crossing the plane placed at $z_{\text {cut }}$ will leave the solid.

Damage to the target caused by energetic ion impacts was studied by calculating local thermodynamic variables, such as temperature, density, hydrodynamic pressure, shear and normal stresses, the coordination number, the slip vector, and the symmetry parameter of the local environment for each atom [43].

\section{SIMULATION RESULTS}

\section{A. HCI interaction with $\mathrm{Si}$ and W}

The silicon and tungsten sputtering yields were calculated for various neutralization times $\tau_{n}=0.1-1000$ fs. The calculated results are shown in Fig. 3 for a highly charged $\mathrm{Xe}^{q+}$ ion, with a kinetic energy of $1 \mathrm{keV}$, bombarding $\mathrm{Si}(100)$ and W (100) surfaces. The experimental results for Si from Ref. [15] are also shown in Fig. 3. Although the recently developed microbalance technique [44] allows one to quantify the surface erosion, no experimental data is available for the sputtering yields of Si surfaces induced by $\mathrm{Xe}^{q+}$ HCIs. The 


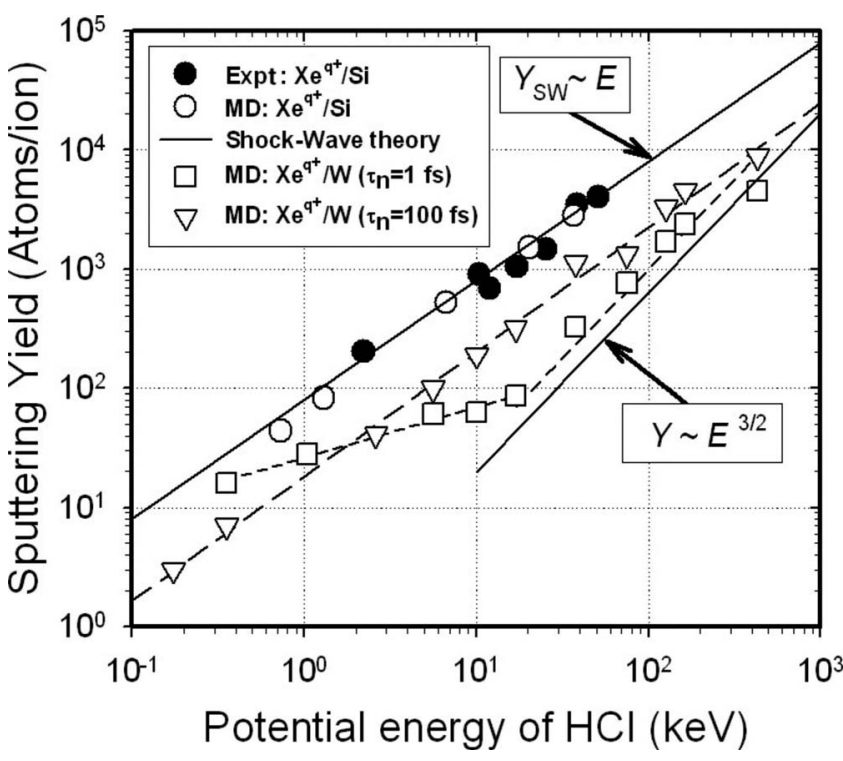

FIG. 3. Comparison of calculated sputtering yield for Si and W surfaces with experimental data available for Si [15]. The dashes are linear fits to the data points and MD data. The tungsten yields are calculated for two neutralization times: $\tau_{n}=1$ and $100 \mathrm{fs}$. The solid lines are drawn according to a simple shock wave theory model [39] and as a 3/2 power law.

calculated data were also compared to the experimental sputtering yields obtained for metal surfaces ( $\mathrm{Al}, \mathrm{Cu}, \mathrm{Ni})$ [15] and $\mathrm{LiF}, \mathrm{SiO}_{2}, \mathrm{GaAs}$ [45], and $\mathrm{UO}_{2}$ [46].

Our comparisons show good agreement of the calculated data with the measurements of the yield from $\mathrm{Si}$ and are the same order of magnitude for all semiconducting materials. For small neutralization times $\left(\tau_{n}<15 \mathrm{fs}\right)$, there are two characteristic energy intervals where the sputtering yields have a small slope below the threshold and a higher, approximately 3/2 power-law dependence above it. The threshold energies for these two energy regions are almost the same for $\mathrm{SiO}_{2}$ and $\mathrm{LiF}$ and are bigger for $\mathrm{W}$ and $\mathrm{UO}_{2}$.

Shock wave generation was studied for a $\mathrm{Xe}^{44+} \mathrm{HCI}$ impact on a Si (100) surface. The upper solid line in Fig. 3 is drawn according to a simple shock wave theory model [39] that predicts a linear dependence of the sputtering yield on the total Coulomb energy.

Two experimental data points are shown in Fig. 4 for both $\mathrm{Cu}$ and $\mathrm{Ni}$ at $2.0-10 \mathrm{keV}$. Above $10 \mathrm{keV}$ the slopes of the broken lines for $\mathrm{Al}, \mathrm{Cu}$, and $\mathrm{Ni}$ become $Y \sim E^{3 / 2}$, which seems more reasonable if we take into account the result for W shown in Fig. 3.

Velocity distribution of the ejected atoms reveals the mechanism of sputtering - the shock wave mechanism gives a $v^{-3}$ dependence at higher velocities, which can be obtained from the shock wave theory $[37,39]$.

A preliminary analysis based on the local atomic stresses and on the slip vector calculation [43] showed that HCI craters strongly emit dislocation loops and stacking faults that are located near the surface and are stable for the whole period of simulation, $75 \mathrm{ps}$. The maximum calculated shear stress for the tungsten target was well above the lattice strength and the tungsten bulk modulus [47]. Such extended

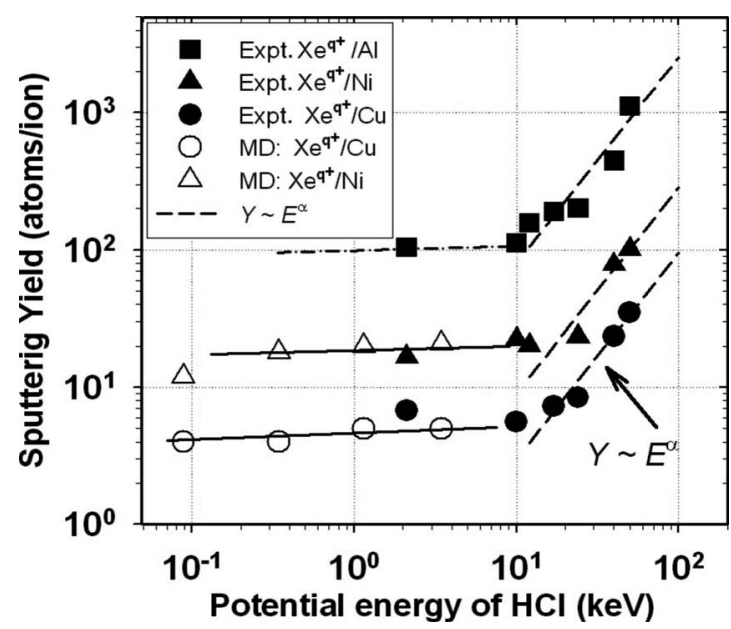

FIG. 4. Comparison of simulation results with experimental data for metals $\mathrm{Al}, \mathrm{Cu}$, and $\mathrm{Ni}$ [15]. The neutralization times for metals are given in Table I. Two data points are shown for both $\mathrm{Cu}$ and $\mathrm{Ni}$ at 2.0 and $10 \mathrm{keV}$. Above $10 \mathrm{keV}$ the slope of the broken lines for $\mathrm{Al}, \mathrm{Cu}$, and $\mathrm{Ni}$ could be changed to become $Y \sim E^{a}$, where $\alpha$ $\approx 3 / 2$ which is reasonable if we take account of the result for $\mathrm{W}$ shown in Fig. 3.

defects can easily be the driving force for the surface hillocks observed on the top of insulating surfaces irradiated by $\mathrm{HCI}$ and by high-energy heavy ions [48-50].

Figure 5 shows two crater shapes obtained by our molecular dynamics simulations for different energies and the same neutralization time. Figure 5(a) shows the crater formed by a $\mathrm{Xe}^{30+}$ ion impact, with a potential energy of $17 \mathrm{keV}$ and a neutralization time of $\tau_{n}=1$ fs. Figure 5(b) shows a larger crater formed by a $\mathrm{Xe}^{44+}$ ion with an energy of $50 \mathrm{keV}$ and a neutralization time of $\tau_{n}=1$ fs.

The main difference between the HCI craters and those produced by conventional heavy ion collisions is that the HCI craters have very small [as in Fig. 5(a)] or no rims [as in Fig. 5(b)] around the crater. This is an important feature that can be used for surface analysis applications with HCI, membrane treatment in biotechnology, and wafer etching in the semiconductor and magnetic storage industries.

Figure 6 shows the shape of the crater simulated on a $\mathrm{Si}$ (100) surface bombarded with a $\mathrm{Xe}^{44+} \mathrm{HCI}$. The rim diameter of the simulated crater shown in Fig. 6 is on the order of $\sim 100 \AA$, which is somewhat smaller than the experimental value of $150 \AA$ measured for a Xe $\mathrm{Xe}^{44+}$ impact on $\mathrm{Si}$ (100) [51]. The discrepancy between these two crater diameters can be related to a possible oxidation of the craters on $\mathrm{Si}$ surfaces in the experiment. The reported Si sputtering yield of Si atoms was on the order of $\sim 100 /$ ion. This yield is two orders of magnitude less than the total number of atoms $\sim 4 \times 10^{4}$ that can fill out a crater with a diameter of $150 \AA$. The only way these two experimental values match is if the crater in the experiment was heavily oxidized after the sputtering.

\section{B. Space charge neutralization in silicon}

The set of Eqs. (7)-(9) was solved numerically at room temperature for the spherical symmetry, and the results are 

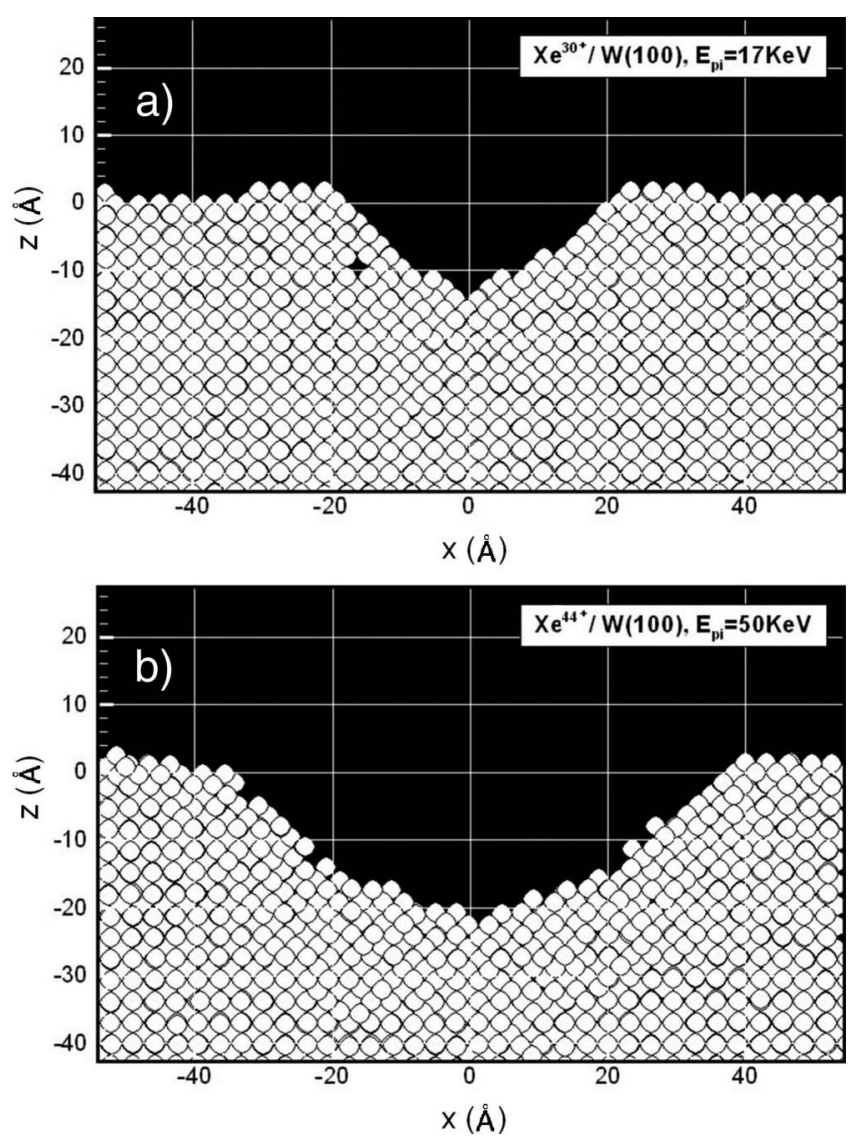

FIG. 5. Two crater shapes obtained by our molecular dynamics simulations. (a): Crater formed by a $\mathrm{Xe}^{30+}$ ion with a potential energy of $17 \mathrm{keV}$ and neutralization time $\tau_{n}=1$ fs. (b) Much shallower crater formed by a $\mathrm{Xe}^{44+}$ ion with an energy of $50 \mathrm{keV}$ and neutralization time $\tau_{n}=1$ fs.

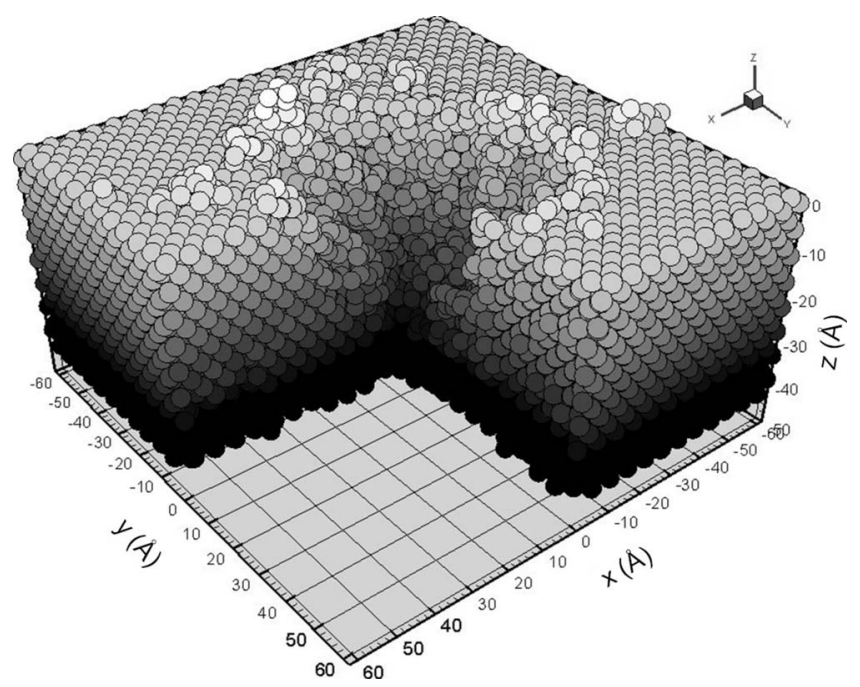

FIG. 6. Shape of the crater formed on a Si (100) surface by bombardment of a $\mathrm{Xe}^{44+} \mathrm{HCI}$. The rim diameter of the simulated crater shown above is on the order of $\sim 100 \AA$, which is less than the experimental value of $150 \AA$ measured for a $\mathrm{Xe}^{44+}$ impact on $\mathrm{Si}$ (100) [51]. The discrepancy between these two crater diameters can be related to a possible oxidation of the craters on Si surfaces in the experiment.

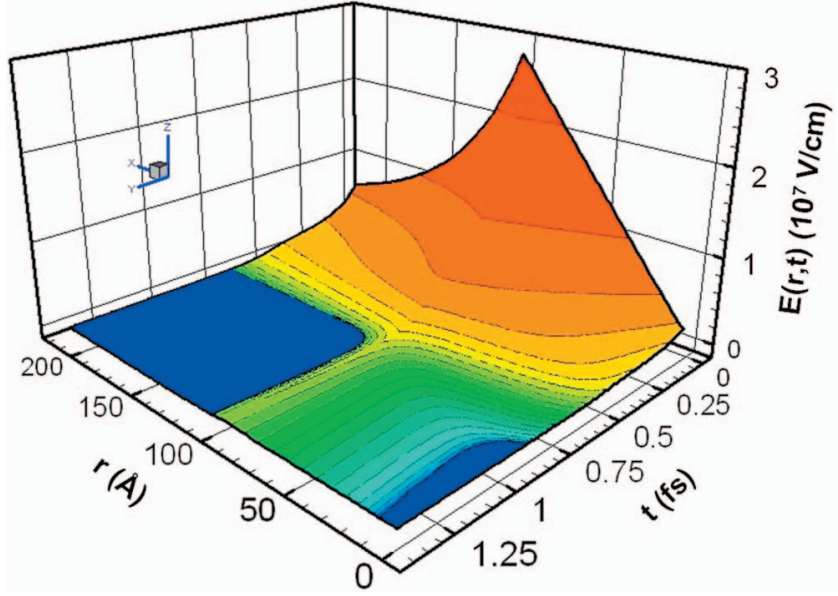

FIG. 7. (Color) Space-time evolution of the electric field during a high energy HCI impact with a Si surface: the space charge neutralization times are obtained by solution of the set of Eqs. (7). The initial variables are $N_{q}=10^{3}$ and $r_{0}=100 \AA$ which correspond to a high HCI energy of $50 \mathrm{keV}$. The Si parameters and transport coefficients used in the calculation are given in Table III.

shown in Figs. 7 and 8 for two different values of induced charges $N_{q}$. At an initial moment, $N_{q}$ charges were inserted into a semispherical region at the surface with a radius of $r_{0}$. The space charge induced by the HCI impact creates an electric field $\boldsymbol{E}$ that is capable of generating impact ionization of
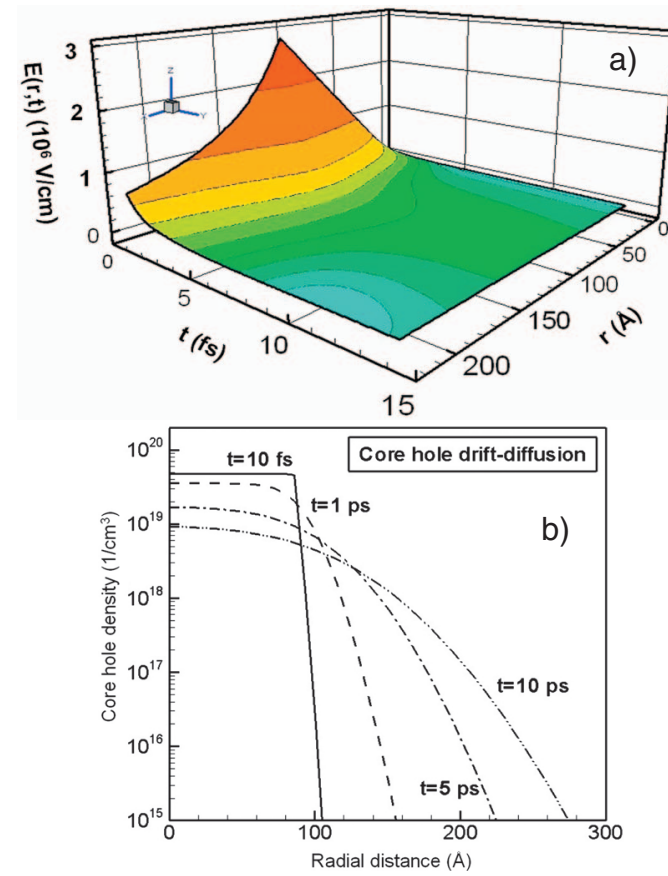

FIG. 8. (Color) (a) Space-time evolution of the electric field and the time-space evolution of the core (heavy) hole density $N_{\mathrm{ch}}$. The initial variables are $N_{q}=100$ and $r_{0}=100 \AA$ which corresponds to a lower HCI energy of $2 \mathrm{keV}$. The Si parameters and transport coefficients used in the calculation are given in Table III. (b) Time and space evolution of the heavy (core) hole density by the drift and diffusion mechanisms at an HCI potential energy of $2 \mathrm{keV}$. The mobility of heavy holes is given in Table III. 
TABLE III. Parameters and transport coefficients of silicon.

\begin{tabular}{ll} 
Silicon atomic density: $n_{0}(\mathrm{Si}) 1 / \mathrm{cm}^{3}$ & $4.99 \times 10^{22}$ \\
Electron diffusivity: $D_{e}, \mathrm{~cm}^{2} / \mathrm{s}$ & 35 \\
Hole diffusivity: $D_{h}, \mathrm{~cm}^{2} / \mathrm{s}$ & 12 \\
Electron mobility: $\mu_{e}, \mathrm{~cm}^{2} / \mathrm{V} \mathrm{s}$ & 1400 \\
Hole mobility: $\mu_{h}=\mathrm{cm}^{2} / \mathrm{V} \mathrm{s}$ & 450 \\
Heavy hole diffusivity: $D_{\mathrm{ch}}, \mathrm{cm}^{2} / \mathrm{s}$ & $1 e-3 \times D h$ \\
Heavy hole mobility: $\mu_{\mathrm{ch}}, \mathrm{cm}^{2} / \mathrm{V} \mathrm{s}$ & $1 e-3 \times \mu_{h}$ \\
Band gap: $E_{g}, \mathrm{eV}$ at $300 \mathrm{~K}$ & 1.1242 \\
Intrinsic carrier density at room temperature: $\mathrm{Ni}$, & $1.3 \times 10^{10}$ \\
$\mathrm{~cm}^{-3}$ & \\
Density of states in conduction band: & $3.22 \times 10^{19}$ \\
Ne, cm & \\
Density of states in valence band: $N v, \mathrm{~cm}^{-3}$ & $1.83 \times 10^{19}$ \\
Auger neutralization times, s $[14,55]$ & $\sim 10^{-14}$ \\
\hline \hline
\end{tabular}

hot electron-hole pairs by accelerating intrinsic charges $N_{i}$. We use the number of induced charges $N_{q}$ and the radius $r_{0}$ as variable parameters and the solution of the set (6)-(8) to define the charge dynamics.

The neutralization times obtained by the plasma model for low potential energy HCI colliding with Si surfaces are comparable to those of the simple model. At a high-energy HCI impact that induces $N_{q} \sim 10^{3}$ electronic charges in a volume with the radius of $r_{0}=100 \AA$, the characteristic times for the electric field change are much shorter (Fig. 7). Such a collision is shown in Figs. 7 and 8 for an impact of a HCI with a Si surface. Figure 7 shows the time and radial distance evolution of the electric field caused by a HCI impact on a $\mathrm{Si}$ surface. Figure 8(a) shows the evolution of the electric field and Fig. 8(b) shows the time and space evolution of the core (heavy) holes. Since the mobilities of heavy holes are unknown, we calculated the set (7) for the following ratios of the mobilities of heavy to light holes $\left(\mu_{\mathrm{ch}} / \mu_{h}=0.1,10^{-2}\right.$, $10^{-3}$ ). The results shown in Fig. 8(b) correspond to the ratio of $10^{-3}$. Since the drift-diffusion of heavy holes is the slowest process in our plasma model, we assume that the neutralization process is finished by the end of the heavy-hole density expansion time. The result in Fig. 8(b) shows that the Maxwell relaxation time (1 ps for silicon) is close to the core hole expansion time.

The characteristic time of two singly ionized atoms separated at a lattice distance $a_{0}$ to move to separation $2 a_{0}$ under Coulomb repulsions $\sim 0.1 \mathrm{ps}$ [6]. This time is slightly lower than the neutralization time given by our models, $1 \mathrm{ps}$. This means that the Coulomb explosion occurs before the neutralization of the space charge takes place. The simulation parameters and transport coefficients of silicon are given in Table III.

The electron-hole plasma generated by a HCI collision with semiconductor and/or insulator surfaces is strongly nonideal and closely resembles the plasma generated by femtosecond laser irradiation [52]. In the density-temperature phase diagram such a plasma corresponds to "warm dense matter" [53]. Figure 9 shows the dependence of the electronhole plasma coupling parameter $\Gamma=\left(4 \pi n_{e} / 3\right)^{1 / 3} e^{2} /\left(k_{B} T_{e}\right)$

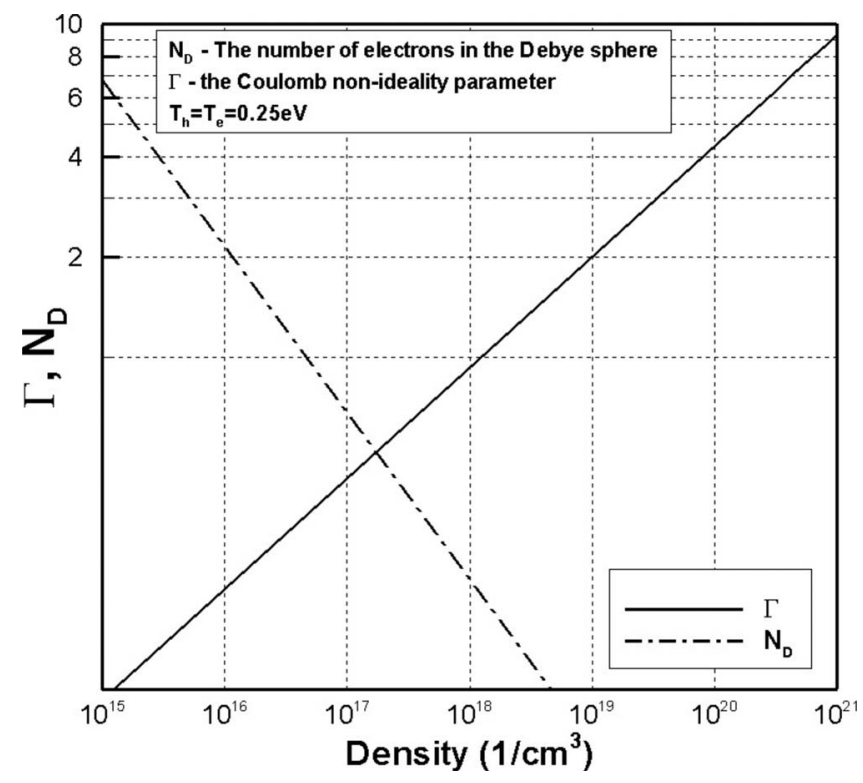

FIG. 9. Dependence of the plasma coupling parameter $\Gamma$ $=\left(4 \pi n_{e} / 3\right)^{1 / 3} e^{2} /\left(k_{B} T_{e}\right)$ and the number of the particles in the Debye sphere $N_{D}=4 \pi r_{D}^{3} n_{e} / 3$, where $r_{D}$ is the radius of the Debye sphere $r_{D}=\sqrt{k_{B} T / 4 \pi n_{e} e^{2}}$.

and the number of the particles in the Debye sphere $N_{D}$ $=4 \pi r_{D}^{3} n_{e} / 3$, where $r_{D}$ is the radius of the Debye sphere $r_{D}$ $=\sqrt{k_{B} T / 4 \pi n_{e} e^{2}}$. The $e$ - $h$ plasma is "hot," with the average temperature of $T_{e, h}=0.25 \mathrm{eV}$ [54].

Figure 9 shows that, at the densities of $10^{17}-10^{18} \mathrm{~cm}^{-3}$, the coupling parameter becomes larger than unity and the number of ions in the Debye sphere becomes less than unity. Such a strongly coupled plasma can successfully be studied by computer simulation methods such as Monte Carlo or molecular dynamics [7].

\section{SUMMARY}

Surface charge neutralization by formation of electronhole plasma was studied, and a simulation model was developed for HCI impacts on a semiconductor surface. The plasma model predicts that the strong electric field of the induced charge is capable of impact ionization of hot electron-hole plasma.

The plasma model predicts the lifetime of a strong electric field $\sim 1$ fs. The hot electron-hole plasma formed by the impact ionization is strongly nonideal and has a lifetime below 1-10 fs. It can therefore be used for numerous applications that need very short times in the range of atto- to femtoseconds, such as quantum dot radiation, defect analysis, and shock wave registration.

Various mechanisms of surface erosion by HCI ion bombardment were studied by the molecular dynamics method: surface erosion due to shock wave generation, crater formation, and sputtering of Si (100) and W (100) surfaces irradiated by highly charged $\mathrm{Xe}^{q+}$ ions $(q=8-44)$.

The diameter of the simulated crater is smaller than the experimental value obtained for a $\mathrm{Xe}^{44+}$ impact on a $\mathrm{Si}(100)$ surface. This result can be explained by possible oxidation of the crater in the experiment. 
The calculated sputtering yields of Si surfaces bombarded by highly charged $\mathrm{Xe}^{q+}$ ions show a good agreement with experiments. We have conducted a preliminary analysis of the stresses and dislocation emission from the impacts of $\mathrm{HCI}$ and accelerated clusters.

The main difference between the HCI craters and those produced by conventional heavy ion collisions is that the HCI craters have a clean crater, with a small rim or no rim at all around the crater. This is an important feature that can be used for surface analysis applications with HCI, membrane treatment in biotechnology, and wafer etching in the semiconductor and magnetic storage industries.

\section{ACKNOWLEDGMENTS}

This work was supported in part by the U.S. Dept. of Energy under Contract No. DE-AC02-06CH11357 and in part by the Grant-In-Aid for Scientific Research for Creative Scientific Research (Grant No. KAKENHI 15GS0217).
[1] J. M. Pomeroy, H. Grube, A. C. Perrella, and J. D. Gillaspy, Appl. Phys. Lett. 91, 073506 (2007).

[2] D. R. Olander, Fundamental Aspects of Nuclear Reactor Fuel Elements (NTIS, U.S. Department of Commerce, Springfield, VA, 1976).

[3] R. L. Kauffman, C. W. Woods, K. A. Jamison, and P. Richard, Phys. Rev. A 11, 872 (1975).

[4] A. Ootuka, K. Kawatsura, F. Fujimoto, K. Komaki, K. Ozawa, and M. Terasawa, J. Phys. Soc. Jpn. 53, 1001 (1984).

[5] D. H. G. Schneider and M. A. Briere, Phys. Scr. 53, 228 (1996).

[6] G. G. Ritchie and C. Claussen, Nucl. Instrum. Methods Phys. Res. 198, 133 (1982).

[7] I. V. Morozov and G. E. Norman, J. Exp. Theor. Phys. 100, 370 (2005).

[8] L. K. Mansur, A. F. Rowcliffe, R. K. Nanstad, S. J. Zinkle, W. R. Corwin, and R. E. Stoller, J. Nucl. Mater. 329-333, 166 (2004).

[9] J. P. Allain, A. Hassanein, T. Burtseva, A. Yacout, Z. Insepov, S. Taj, and B. J. Rice, Proc. SPIE 5374, 112 (2004).

[10] A. Hamza, T. Schenkel, A. Barnes, and D. Schneider, U.S. Patent No. 6,261,820 B1 (18 Sept. 2001).

[11] C. Ruehlicke, D. Schneider, M. Schneider, R. D. DuBois, and R. Balhorn, Nanotechnology 9, 251 (1998).

[12] P. Sigmund, Phys. Rev. 184, 383 (1969).

[13] H. Winter and F. Aumayr, J. Phys. B 32, R39 (1999).

[14] J. Burgdörfer, P. Lerner, and F. W. Meyer, Phys. Rev. A 44, 5674 (1991); J. Burgdörfer, in Fundamental Processes and Applications of Atoms and Ions, edited by C. D. Lin (World Scientific, Singapore, 1993), p. 517.

[15] T. Sekioka, M. Terasawa, T. Mitamura, M. P. Stockli, U. Lehnert, and C. Fehrenbach, Nucl. Instrum. Methods Phys. Res. B 146, 172 (1998); 182, 121 (2001).

[16] H. Winter and F. Aumayr, J. Phys. B 32, R39 (1999).

[17] K. Tökési, L. Wirtz, C. Lemell, and J. Burgdörfer, Phys. Rev. A 64, 042902 (2001).

[18] E. M. Bringa and R. E. Johnson, Phys. Rev. Lett. 88, 165501 (2002).

[19] A. H. Marshak, Proc. IEEE 72, 148 (1984).

[20] A. G. Chynoweth, J. Appl. Phys. 31, 1161 (1960).

[21] R. Van Overstraeten, Solid-State Electron. 13, 583 (1970).

[22] L. M. Biberman, I. T. Yakubov, and V. S. Vorob'ev, Proc. IEEE 59, 555 (1972).

[23] A. Paskin and G. J. Dienes, J. Appl. Phys. 43, 1605 (1972).

[24] V. Yu. Klimenko and A. N. Dremin, Sov. Phys. Dokl. 25, 288
(1980).

[25] B. L. Holian, W. G. Hoover, B. Moran, and G. K. Straub, Phys. Rev. A 22, 2798 (1980).

[26] B. L. Holian, in Microscopic Simulations of Complex Hydrodynamic Phenomena, edited by M. Mareschal and B. L. Holian, NATO ASI Series B (Plenum, New York, 1993), Vol. 292, p. 75.

[27] R. P. Webb and D. E. Harrison, Jr., Appl. Phys. Lett. 39, 311 (1981).

[28] U. Even, I. Shek, and J. Jortner, Chem. Phys. Lett. 202, 303 (1993).

[29] Z. Insepov and I. Yamada, Nucl. Instrum. Methods Phys. Res. B 112, 16 (1996).

[30] R. Aderjan and H. M. Urbassek, Nucl. Instrum. Methods Phys. Res. B 164, 697 (2000).

[31] C. Anders, H. M. Urbassek, and R. E. Johnson, Phys. Rev. B 70, 155404 (2004).

[32] E. M. Bringa and R. E. Johnson, Nucl. Instrum. Methods Phys. Res. B 143, 513 (1998).

[33] E. M. Bringa, R. E. Johnson, and L. Dutkiewicz, Nucl. Instrum. Methods Phys. Res. B 152, 267 (1999).

[34] E. M. Bringa, K. Nordlund, and J. Keinonen, Phys. Rev. B 64, 235426 (2001).

[35] E. M. Bringa, R. E. Johnson, and R. M. Papaleo, Phys. Rev. B 65, 094113 (2002).

[36] M. M. Jakas, E. M. Bringa, and R. E. Johnson, Phys. Rev. B 65, 165425 (2002).

[37] Z. Insepov and I. Yamada, Nucl. Instrum. Methods Phys. Res. B 121, 44 (1997); Z. Insepov, R. Manory, J. Matsuo, and I. Yamada, Phys. Rev. B 61, 8744 (2000).

[38] L. Verlet, Phys. Rev. 159, 98 (1967); E. Egami, K. Maeda, and V. Vitek, Philos. Mag. A 41, 883 (1980).

[39] Ya. B. Zel'dovich and Yu. P. Raizer, Physics of Shock Waves and High Temperature Hydrodynamic Phenomena (Academic Press, New York, 1967).

[40] F. H. Stillinger and T. A. Weber, Phys. Rev. B 31, 5262 (1985).

[41] L. V. Woodcock, C. A. Angell, and P. Cheesemann, J. Chem. Phys. 65, 1565 (1976).

[42] M. Finnis and J. Sinclair, Philos. Mag. A 50, 45 (1984); G. J. Ackland and R. Thetford, ibid. 56, 15 (1987).

[43] C. L. Kelchner, S. J. Plimpton, and J. C. Hamilton, Phys. Rev. B 58, 11085 (1998); J. A. Zimmerman, C. L. Kelchner, P. A. Klein, J. C. Hamilton, and S. M. Foiles, Phys. Rev. Lett. 87, 165507 (2001) 
[44] F. Aumayr, P. Varga, and H. P. Winter, Int. J. Mass Spectrom. 192, 415 (1999).

[45] T. Schenkel, A. V. Hamza, A. V. Barnes, D. H. Schneider, J. C. Banks, and B. L. Doyle, Phys. Rev. Lett. 81, 2590 (1998).

[46] T. Schenkel, A. V. Barnes, A. V. Hamza, D. H. Schneider, J. C. Banks, and B. L. Doyle, Phys. Rev. Lett. 80, 4325 (1998).

[47] D. Roundy, C. R. Krenn, M. L. Cohen, and J. W. Morris, Jr., Philos. Mag. A 81, 1725 (2001).

[48] I. C. Gebeshuber, S. Cerusca, F. Aumayr, and H. P. Winter, Nucl. Instrum. Methods Phys. Res. B 205, 751 (2003).

[49] I. C. Gebeshuber, S. Cerusca, F. Aumayr, and H. P. Winter, Int. J. Mass Spectrom. 229, 27 (2003).
[50] V. A. Skuratov, S. J. Zinkle, A. E. Efimov, and K. Havancsak, Surf. Coat. Technol. 196, 56 (2005).

[51] A. V. Hamza, M. W. Newman, P. A. Thielen, and H. W. H. Lee, Appl. Phys. Lett. 79, 2973 (2001).

[52] J. Bonse, S. Baudach, J. Krüger, W. Kautek, and M. Lenzner, Appl. Phys. A: Mater. Sci. Process. 74, 19 (2002).

[53] M. Koenig et al., Plasma Phys. Contr. Fusion 47, B441 (2005).

[54] P. A. Wolff, J. Phys. Chem. Solids 16, 184 (1960).

[55] P. Palmeri, P. Quinet, N. Zitane, and N. Vaeck, J. Phys. B 34, 4125 (2001). 